\title{
CHILDBIRTH EXPERIENCE DURING THE COVID-19 PANDEMIC IN MATERNITY CARE INSTITUTIONS IN LATVIA
}

\author{
Sabine Kovale \\ Riga Stradins University, Latvia \\ Madara Teraudkalna \\ Riga Stradins University, Latvia \\ Beate Vasioleka \\ Riga Stradins University, Latvia \\ Kristine Rasnaca \\ Riga Stradins University; Riga Maternity Hospital; \\ Children’s Clinical University Hospital, Latvia
}

\begin{abstract}
The COVID-19 pandemic has had a major impact on people's lives worldwide, causing stress and anxiety for many people, including pregnant women. In addition do not knowing the implications of the new disease, pregnant women may also be negatively impacted by public health actions, such as limitations for accompanying persons to participate in childbirth and overall social distancing. The main objective of the research is to compare childbirth among women who gave birth during the COVID-19 pandemic with a control group. The data was collected using a questionnaire for Assessing the Childbirth Experience. 537 women who gave birth in 2019 and 2020 were included in the research and divided into two groups based on whether they gave birth before or after the state of emergency was declared in Latvia on March 12, 2020. The data was analysed using the IBM SPSS statistics program. The results showed that the childbirth experience for women giving birth without an accompanying person was statistically inferior on several accounts (process control, level of fear, breastfeeding support, etc.) than for women who had an accompanying person participating. In the research group there were proportionally more such women than in the control group, showing that COVID-19 has negatively impacted childbirth experience for many women.
\end{abstract}

Keywords: Childbirth experience; COVID-19; maternity care; pandemic.

\section{Introduction}

The World Health Organization (WHO) announced the global coronavirus disease 2019 (COVID-19) a pandemic, in March 2020 (World Health Organization [WHO], 2020). The COVID-19 outbreak has led to the death of over two million people leaving a huge impact on health care institutions worldwide 
(WHO, 2021). Due to the high transmission rate of COVID-19, society had to drastically change its daily habits, practice social distancing and intensify hygiene procedures. In Latvia the state of emergency was declared on March 12, 2020 (Ministry of Culture of the Republic of Latvia, 2020). Following the state of emergency declaration in Latvia, maternity care institutions had to implement restrictions, which varied depending on the maternity care institution, such as prohibit an accompanying person in birth, cancel maternity preparation courses, place women with upper respiratory tract symptoms in airborne infection isolation rooms until the infection with COVID-19 is ruled out (Riga Maternity Hospital, 2020). Pregnant women are already more susceptible to infections due to the immune system natural suppression (Jamieson, Theiler, \& Rasmussen, 2006). Furthermore, both the pregnancy itself and the lack of information about the effects of COVID-19 on the foetus can result in increased psychological stress and have a negative effect on pregnancy, childbirth and postpartum stages (Traylor, Johnson, Kimmel, \& Manuck, 2020). Elevated stress levels can increase the risk of postpartum blues and depression, difficulties bonding with the newborn, increased negative feelings towards the baby (Bell, Assistant, Andersson, \& Senior, 2016), complications with breastfeeding (Beck \& Watson, 2008) and also fear of future deliveries. Therefore, the objective of this study was to compare childbirth and the early neonatal period experience for mothers before and after the state of emergency was declared in Latvia on March 12, 2020 due to the COVID-19 pandemic. As many efforts to protect the medical staff and patients from the infection with COVID-19 were made, we hypothesized that the distress caused by the pandemic as well as maternity care institutions restrictions directed at reducing the transmission rate could be associated with lower assessment of childbirth and the early neonatal period experience for mothers.

\section{Literature Review}

Increased levels of stress during pregnancy and childbirth have been recognized to have a negative effect on both pregnancy outcomes as well as mental health of the mother post delivery. Research shows that stressful changes in life during pregnancy are associated with poorer pregnancy outcomes (Williamson, Le Fevre, \& Hector, 1989), higher rates of instrumental deliveries (Romero-Gonzalez, Caparros-Gonzalez, Gonzalez-Perez, Coca-Arco, \& PeraltaRamirez, 2019) as well as can have a significant contribution in development of postpartum mood disorders (Kingston, Tough, \& Whitfield, 2012). Elevated levels of stress during labour also have been shown to affect the mother's mental health and have been associated with increased risk for development of childbirthrelated posttraumatic stress disorder (Chan et al., 2020). Such disorder can further 
lead to problems of maternal bonding and breastfeeding of the newborn (Dekel, Thiel, Dishy, \& Ashenfarb, 2019).

It is not surprising, that in recent studies also the COVID-19 pandemic has been shown to have an adverse effect on the pregnancy, childbirth and postpartum period. Several studies have shown association between the pandemic and poorer antenatal care - lower number and delay of antenatal visits (Temesgen, Workie \& Dilnessa, 2020; Goyal et al., 2020). Since the outbreak of COVID-19, research has been done to assess the effects on maternal mental health. Elevated depression, anxiety and post-traumatic stress disorder symptoms have been reported among women who gave birth during the COVID-19 pandemic (Wu et al., 2020; Ostacoli et al., 2020; Marino-Narvaez, Puertas-Gonzalez, RomeroGonzalez, \& Peralta-Ramirez, 2020).

The research on women's experience of childbirth during the COVID-19 pandemic is rather scarce. One research showed that childbirth during the COVID-19 pandemic is associated with higher perceived stress levels and poorer quality of care during delivery (Marino-Narvaez et al., 2020). Another research also showed that women delivering during the COVID-19 pandemic had a significantly higher acute stress response than the sociodemographically matched control group. Furthermore, it was shown that higher acute stress response during delivery is associated with elevated post-traumatic stress disorder symptoms and worse maternal bonding with the infant (Mayopoulos et al., 2021).

The COVID-19 pandemic as a stressor affects not only the women giving birth, but also the healthcare professionals providing care during delivery. The pandemic has been associated with higher levels of distress in health workers especially in those who work with COVID-19 positive patients (Babore et al., 2020). This in turn might have an impact on the women's experience of childbirth, as high levels of stress, burn-out and depressive symptoms can affect a health professional's ability to provide high quality of care (Koinis et al., 2015).

In addition to the stress from not knowing the implications of the new disease, the women giving birth during pandemic may also be negatively impacted by public health actions, such as limitations for accompanying persons to participate in childbirth and overall social distancing. It has been shown that the father of the child can provide emotional support during delivery and postpartum (Firouzan, Noroozi, Mirghafourvand, \& Farajzadegan, 2018).

\section{Methodology}

\section{Participants and setting}

A total of 537 women participated in a cross-sectional postnatal online questionnaire study. Women were identified in the postnatal period and offered 
to enter the study. Those who agreed to join completed the questionnaire via an online survey tool. The inclusion criteria were to be of legal age (at least 18 years old), to be able to read and write in Latvian and to have given birth between January 2019 and December 2020 in Latvian maternity care institutions.

All participants were divided into two groups: a research group (women who gave birth after the state of emergency was declared in Latvia) and a control group (women who gave birth before the state of emergency in Latvia).

\section{Instruments}

First of all, sociodemographic (age, level of education, place of residence, etc.) data was obtained from the participants, as well as obstetric variables (gestational age at birth, delivery mode, number of births, complications, participation of a support person, use of epidural anaesthesia, etc.) and the early newborn period (admission to neonatal intensive care unit). Secondly an evaluation was conducted using evaluation instruments described below.

\section{Childbirth experience questionnaire (CEQ)}

The CEQ was translated and adapted from English to Latvian. The CEQ has 21 questions assessing four main domains of childbirth experience (Dencker et al., 2010). Women's responses for 18 from the 21 statements are rated on a 4-point Likert Scale. Questions about labour pain, sense of control and security are assessed with visual analogue scales (VAS), see Table 1. The higher the score, the better the birth experience. Ratings of negatively worded statements are reversed.

Table 1 Childbirth Experience Questionnaire (CEQ) Domains and Included Items

\begin{tabular}{lcl}
\hline Domain & Items & \\
\hline Own capacity & - & Labour and birth went as I had expected. \\
& - & I felt strong during labour and birth. \\
& - & I felt capable during labour and birth. \\
& - & I was tired during labour and birth. \\
& - & I felt happy during labour and birth. \\
& - & I felt that I handled the situation well. \\
& - & As a whole, how painful did you feel childbirth was? $*$ \\
& - & As a whole, how much control did you feel you had during \\
support & & childbirth? $*$ \\
\hline Profsional & - & Both my partner and I were treated with warmth and respect. \\
& - & My midwife/doctor devoted enough time to me and my partner. \\
& - & My midwife/doctor kept me informed about what was happening \\
& - & during labour and birth. \\
& - & I felt very well cared for by my midwife/ doctor. \\
\hline
\end{tabular}




\begin{tabular}{|c|c|}
\hline $\begin{array}{l}\text { Perceived } \\
\text { safety }\end{array}$ & $\begin{array}{l}\text { - } \quad \text { I felt scared during labour and birth. } \\
\text { - } \quad \text { My impression of the team's medical skills made me feel secure. } \\
\text { - } \quad \text { I have many positive memories from childbirth. } \\
\text { - } \quad \text { I have many negative memories from childbirth. } \\
\text { - } \quad \text { Some of my memories from childbirth make me feel depressed. } \\
\text { - }\end{array}$ \\
\hline Participation & $\begin{array}{l}\text { - } \quad \text { I felt I could have a say in deciding my birthing position. } \\
\text { - } \quad \text { I felt I could have a say in the choice of pain relief. }\end{array}$ \\
\hline
\end{tabular}

*VAS-scale with anchors.

We also included questions about the early newborn period, which is defined as age 0-6 days, to supplement the results obtained from the CEQ. The section about the early newborn period included 9 statements with a response format of a 4-point Likert scale, see Table 2.

\section{Table 2 Early Newborn Period Section of the Questionnaire}

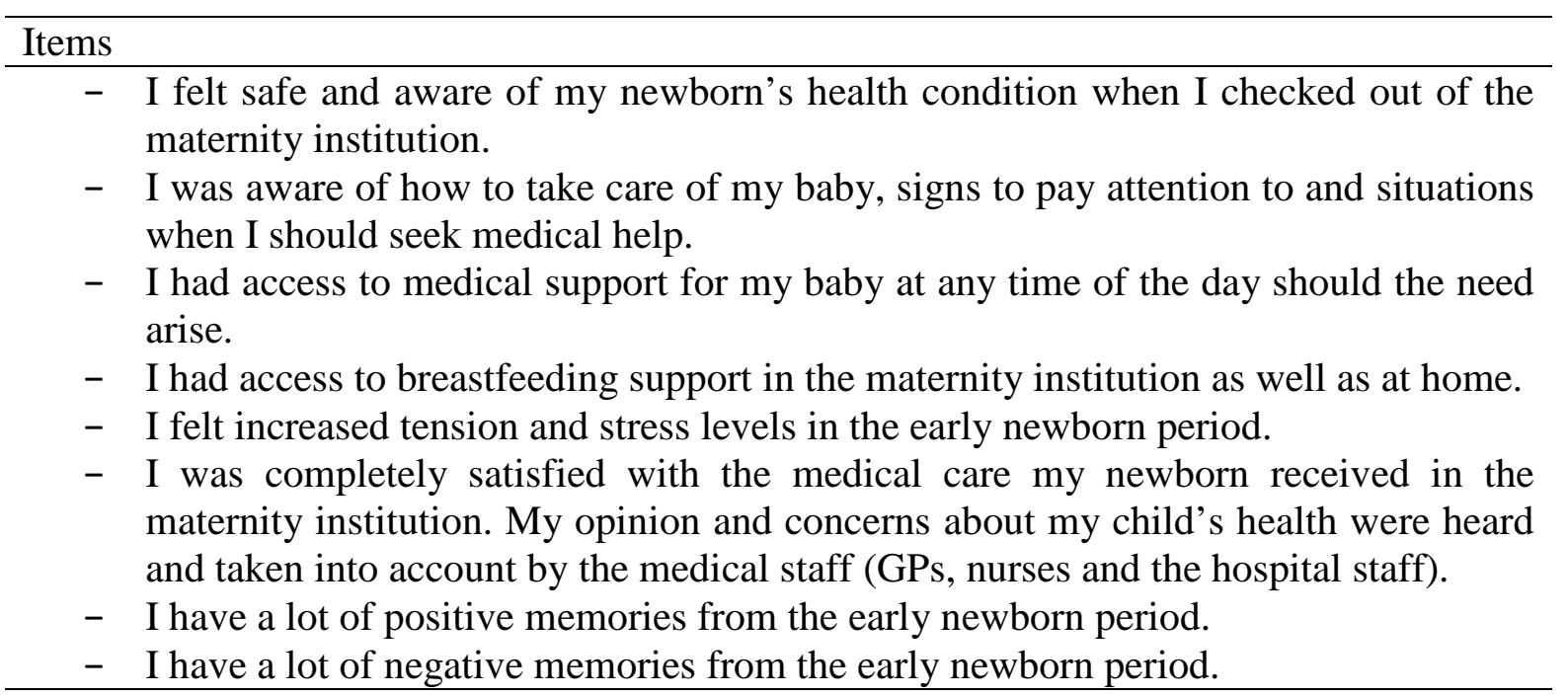

\section{Ethics}

All participants voluntarily entered the study. Informed consent was obtained. Anonymity and confidentiality of the data obtained was ensured. The protocol was approved by the Riga Stradins University (RSU) Research Ethics Committee (reference code 6-1/13/2020/9).

\section{Statistics and Data Analysis}

The data was analysed using IBM SPSS for Windows, Version 26.0. Continuous variables are expressed as the mean \pm standard deviation (SD), while categorical variables are expressed as frequency and percentage. Sociodemographic and obstetric characteristics were compared between groups 
using Student t tests (means) for normally distributed continuous variables and chi-squared tests (\%) for categorical variables. Mann - Whitney U test was used to compare skewed continuous variables and nominal variables. $\mathrm{P}$ value $<0.05$ was considered as statistically significant. A 95\% confidence level was used.

\section{Research Results}

A total of 537 women fulfilled the inclusion criteria and were divided into two groups (research group $n=311$ and control group $n=216$ ) based on whether they gave birth before or after the state of emergency due to the COVID-19 pandemic was declared in Latvia. The key sociodemographic and birth related factors have been summarized in Table 3. Overall, there were no statistically significant differences between two groups based on sociodemographic and obstetric factors, except for planning and actual participation of a support person in childbirth. In the research group, a statistically higher proportion of women during pregnancy were planning for a support person to participate in childbirth if compared with the control group (88.4\% vs $80.1 \%$ : Pearson Chi-square value $7.101, \mathrm{p}=0.008$ ). Despite that, the actual participation rate of a support person was significantly lower in the research group, compared to the control group (62.7\% versus 74.8\%; Pearson Chi-square value $8.743, \mathrm{p}=0.003$ ). Furthermore, overall women that planned for participation of a support person in childbirth during pregnancy but were giving birth without the participation of a support person $(n=94)$ reported inferior childbirth experience on several accounts if compared with women who planned and had support person participating $(n=362)$. These accounts include the control level of the process $(U=14280.50, p=0.016)$, the level of fear $(\mathrm{U}=14706.50, \mathrm{p}=0.036)$, the level of how one's choice regarding pain management was taken into account $(\mathrm{U}=14716, \mathrm{p}=0.016)$ and level of breastfeeding support $(\mathrm{U}=13681, \mathrm{p}=0.002)$.

If overall childbirth experience and early newborn period experience is compared between research and control groups, then a statistically significant difference is observed in only one aspect out of a total of 30 and that is - that my midwife/doctor understood my needs and listened to me. In the research group the median value of the variable was 4 , while in the control group 3 ( $U=38325.5$, $\mathrm{p}=0.049$ ).

If subgroups of research and control groups based on different obstetric factors are compared, the following results are obtained.

For nulliparous women there is a statistically significant difference in mean ranks of variable - I was aware of how to take care of my baby, signs to pay attention to and situations when I should seek medical help $(\mathrm{U}=8530, \mathrm{p}=0.013)$ with the research group $(\mathrm{n}=152)$ having lower mean rank than the control group $(n=134)$. 
Among women who gave birth via caesarean there were statistically significant differences between the research and the control group on several accounts:

- My midwife/doctor devoted enough time to me and my partner $(\mathrm{U}=1508.50, \mathrm{p}=0.035)$

- I felt very well cared for by my midwife/ doctor $(U=1538.00, p=0.018)$

- I have many negative memories from childbirth $(U=953.00, p=0.039)$

- Some of my memories from childbirth make me feel depressed $(\mathrm{U}=934.00, \mathrm{p}=0.028)$

For the first two aspects the median values were higher in the research group than in the control group, respectively 4 versus 3 and 4 versus 3.5, while for latter two aspects, the mean ranks were lower in the research group, compared with the controls group. Among women who gave birth vaginally there were no statistical differences in the mean ranks of any variables, as well as there were also no statistical differences among multipara women.

Table 3 Summary of Sociodemographic and Obstetric Factors

\begin{tabular}{|c|c|c|c|}
\hline Factor & $\begin{array}{l}\text { Research group } \\
(\mathrm{n}=311)\end{array}$ & $\begin{array}{l}\text { Control group } \\
(\mathrm{n}=226)\end{array}$ & $P$ value \\
\hline \multicolumn{4}{|l|}{ Sociodemographic } \\
\hline $\begin{aligned} & \text { - Age } \\
&-\quad \text { Education } \\
&- \text { Primary } \\
&- \text { Secondary } \\
&- \text { Higher }\end{aligned}$ & $\begin{array}{l}29.51 \pm 4.856 \\
1.3 \% \\
32.2 \% \\
66.2 \%\end{array}$ & $\begin{array}{l}29.06 \pm 4,782 \\
2.2 \% \\
27.4 \% \\
70.4 \%\end{array}$ & $\begin{array}{l}0.289 \\
0.442\end{array}$ \\
\hline \multicolumn{4}{|l|}{ Obstetric } \\
\hline 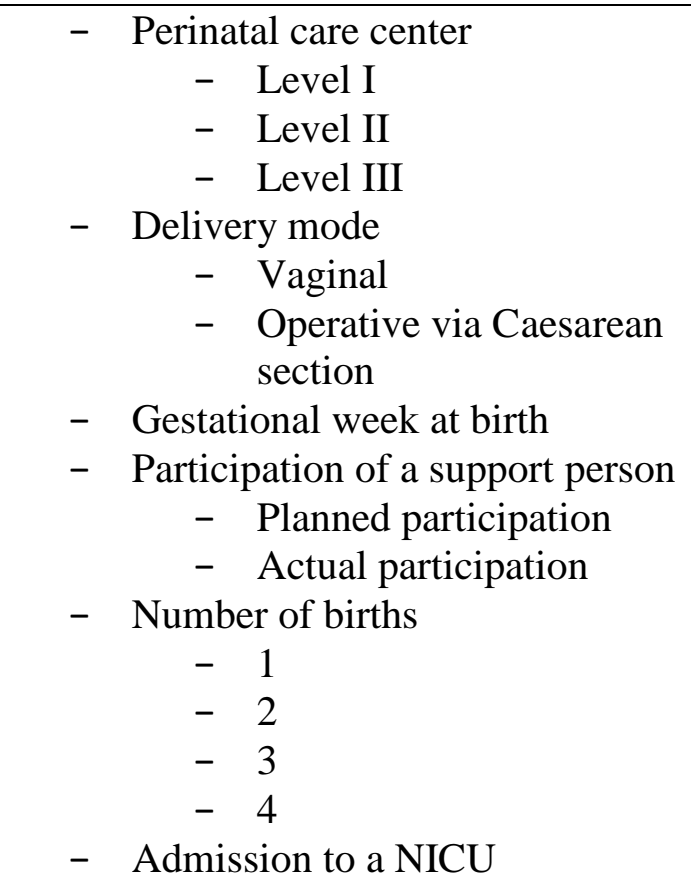 & $\begin{array}{l}43.4 \% \\
47.3 \% \\
9.3 \% \\
80.1 \% \\
19.9 \% \\
39.57 \pm 1,477 \\
88.4 \% \\
62.7 \% \\
48.9 \% \\
38.6 \% \\
10.6 \% \\
1.9 \% \\
8.0 \%\end{array}$ & $\begin{array}{l}45.1 \% \\
46.9 \% \\
8.0 \% \\
\\
82.3 \% \\
17.7 \% \\
\\
39.58 \pm 1,688 \\
\\
70.1 \% \\
74.8 \% \\
59.6 \% \\
29.3 \% \\
9.8 \% \\
1.3 \% \\
7.1 \%\end{array}$ & $\begin{array}{l}0.500 \\
0.008 \\
\mathbf{0 . 0 0 3} \\
0.095\end{array}$ \\
\hline
\end{tabular}




\section{Discussion}

This study provides new insight on women's experience giving birth during a global pandemic. We found that during the COVID-19 pandemic, statistically significantly more women planned for a support person's participation in childbirth compared with the control group. This might be explained by distress and fear related to the new pandemic. Recent research shows that due to COVID19 pregnant women experience increased levels of anxiety (Saccone et al., 2020), which might lead to even changing the birth plan away from delivering in hospital (Moyer, Compton, Kaselitz, \& Muzik, 2020). However, due to the state of emergency and subsequent limitations for a support person participation in childbirth, an actual rate of a support person's participation in childbirth was statistically significantly lower than before pandemic. A support person's participation has been shown to be an important factor that contributes to overall childbirth, leading to fewer negative feelings about childbirth experience, shorter labour, lower frequency of both caesarean and instrumental vaginal delivery (Bohren, Hofmeyr, Sakala, Fukuzawa, \& Cuthbert, 2013). Assistance provided by a support person facilitates women to feel safe, strong, confident and secure (Bohren, Berger, Munthe-Kaas, \& Tuncalp, 2019). This is also supported by our results, which show that women giving birth without the participation of a support person, although they planned for it during pregnancy, have reported inferior childbirth experience with regard to several aspects, including control level of the process, the level of fear, the level of how one's choice regarding pain management was taken into account and the level of breastfeeding support.

Marino-Narvaez et al. in their research found that women who gave birth during the COVID-19 pandemic in Spain reported lower quality of care if compared with women who gave birth before the pandemic (Marino-Narvaez et al., 2020). However, our research shows that despite the elevated proportion of women who had to give birth without the participation of a support person, the overall birth experience and provided care was not significantly inferior for women giving birth during the COVID-19 pandemic if compared with the control group. On the contrary, the women who gave birth during the COVID-19 pandemic reported a higher median value of a variable which accounts for how well the doctor/midwife listened and understood one's needs. This might show that during the COVID-19 pandemic medical personnel involved in the care of labouring mothers, have paid more attention in order to compensate for absence of a support person in childbirth. It may be especially true in case of a more complicated childbirth as our results shows that women who had delivery via caesarean section during the COVID-19 pandemic reported higher median values of both the time allotted to them by doctor/midwife and the level of care provided by doctor/midwife if compared with the respective control group. While in case 
of vaginal delivery there were no statistically significant differences between the two groups.

Our results show that not only childbirth experience might be adversely affected by the pandemic, but also the early postpartum period. Nulliparous women reported a statistically significantly lower mean rank of the variable about knowledge of how to take care of a baby, signs to pay attention to and situations when to seek medical help more than nulliparous women in the control group. This could be partially explained by a more scrupulous infectious control, which in turn might limit the time and possibility for healthcare professionals to educate mothers on such aspects.

\section{References}

Babore, A., Lombardi, L., Viceconti, M. L., Pignataro, S., Marino, V., Crudele, M., Candelori, C., Bramanti, S. M., \& Trumello, C. (2020). Psychological effects of the COVID-2019 pandemic: Perceived stress and coping strategies among healthcare professionals. Psychiatry Research, 293(May), 113366. https://doi.org/10.1016/j.psychres.2020. 113366

Beck, C. T., \& Watson, S. (2008). Impact of birth trauma on breast-feeding: a tale of two pathways. Nursing research, 57(4), 228-236. DOI: 10.1097/01.NNR.0000313494.87282.90.

Bell, A. F., Assistant, C. N. M., Andersson, E., \& Senior, R. N. M. (2016). The birth experience and women's postnatal depression: A systematic review. Midwifery, 39, 112-123. https://doi.org/10.1016/j.midw.2016.04.014)

Bohren, M.A., Hofmeyr G.J., Sakala C., Fukuzawa R.K., Cuthbert A. (2013). Continuous support for women during childbirth. Cochrane Database of Systematic Reviews, 2013(7). https://doi.org/10.1002/14651858.CD003766.pub5

Bohren, M.A., Berger, B.O., Munthe-Kaas H, Tuncalp O. (2019). Perceptions and experiences of labour companionship: a qualitative evidence synthesis (Review). Cochrane Database of Systematic Reviews 2019(3). DOI: 10.1002/14651858.CD012449.pub2

Chan, S. J., Ein-Dor, T., Mayopoulos, P. A., Mesa, M. M., Sunda, R. M., McCarthy, B. F., Kaimal, A. J., \& Dekel, S. (2020). Risk factors for developing posttraumatic stress disorder following childbirth. Psychiatry Research, 290(May), 113090. https://doi.org/10.1016/j.psychres.2020.113090

Dekel, S., Thiel, F., Dishy, G., \& Ashenfarb, A. L. (2019). Is childbirth-induced PTSD associated with low maternal attachment? Archives of Women's Mental Health, 22(1), 119-122. https://doi.org/10.1007/s00737-018-0853-y

Dencker, A., Taft, C., Bergqvist, L. et al. (2010). Childbirth experience questionnaire (CEQ): development and evaluation of a multidimensional instrument. BMC Pregnancy Childbirth 10, 81. https://doi.org/10.1186/1471-2393-10-81

Firouzan, V., Noroozi, M., Mirghafourvand, M., \& Farajzadegan, Z. (2018). Participation of father in perinatal care: A qualitative study from the perspective of mothers, fathers, caregivers, managers and policymakers in Iran. BMC Pregnancy and Childbirth, 18(1), 1-10. https://doi.org/10.1186/s12884-018-1928-5

Goyal, M., Singh, P., Singh, K., Shekhar, S., Agrawal, N., \& Misra, S. (2020). The effect of the COVID-19 pandemic on maternal health due to delay in seeking health care: Experience 
from a tertiary center. International Journal of Gynecology and Obstetrics, October, 15. https://doi.org/10.1002/ijgo.13457

Jamieson, D. J., Theiler, R. N., \& Rasmussen, S. A. (2006). Emerging Infections and Pregnancy, 12(11). Retrieved from: www.cdc.gov/eid

Kingston, D., Tough, S., \& Whitfield, H. (2012). Prenatal and postpartum maternal psychological distress and infant development: A systematic review. Child Psychiatry and Human Development, 43(5), 683-714. https://doi.org/10.1007/s10578-012-0291-4

Koinis, A., Giannou, V., Drantaki, V., Angelaina, S., Stratou, E., \& Saridi, M. (2015). The impact of healthcare workers job environment on their mental-emotional health. Coping strategies: the case of a local general hospital. Health Psychology Research, 3(1). https://doi.org/10.4081/hpr.2015.1984

Marino-Narvaez, C., Puertas-Gonzalez, J. A., Romero-Gonzalez, B., \& Peralta-Ramirez, M. I. (2020). Giving birth during the COVID-19 pandemic: The impact on birth satisfaction and postpartum depression. International Journal of Gynecology \& Obstetrics, 18. https://doi.org/10.1002/ijgo.13565

Mayopoulos, G. A., Ein-Dor, T., Dishy, G. A., Nandru, R., Chan, S. J., Hanley, L. E., Kaimal, A. J., \& Dekel, S. (2021). COVID-19 is associated with traumatic childbirth and subsequent mother-infant bonding problems. Journal of Affective Disorders, 282(December 2020), 122-125. https://doi.org/10.1016/j.jad.2020.12.101

Ministry of Culture of the Republic of Latvia. (2020). On the Declaration of Emergency Situation. Retrieved from: https://www.km.gov.lv/en/article/declaration-emergencysituation

Moyer, C. A., Compton, S. D., Kaselitz, E., \& Muzik, M. (2020). Pregnancy-related anxiety during COVID-19: a nationwide survey of 2740 pregnant women. Archives of Women's Mental Health. https://doi.org/10.1007/s00737-020-01073-5

Ostacoli, L., Cosma, S., Bevilacqua, F., Berchialla, P., Bovetti, M., Carosso, A. R., Malandrone, F., Carletto, S., \& Benedetto, C. (2020). Psychosocial factors associated with postpartum psychological distress during the Covid-19 pandemic: a cross-sectional study. BMC Pregnancy and Childbirth, 20(1), 1-9. https://doi.org/10.1186/s12884-020-03399-5

Riga Maternity Hospital. (2020). During the COVID-19 pandemic, restrictions have been imposed in the Riga Maternity Hospital. Retrieved from: https://www.rdn.lv/en/news/during-the-covid-19-pandemic-restrictions-have-beenimposed-in-the-riga-maternity-hospital-14005/

Romero-Gonzalez, B., Caparros-Gonzalez, R. A., Gonzalez-Perez, R., Coca-Arco, S., \& Peralta-Ramirez, M. I. (2019). Hair cortisol levels, psychological stress and psychopathological symptoms prior to instrumental deliveries. Midwifery, 77, 45-52. https://doi.org/10.1016/j.midw.2019.06.015

Saccone, G., Florio, A., Aiello, F., Venturella, R., De Angelis, M. C., Locci, M., Bifulco, G., Zullo, F., \& Di Spiezio Sardo, A. (2020). Psychological impact of coronavirus disease 2019 in pregnant women. American Journal of Obstetrics and Gynecology, 223(2), 293295. https://doi.org/10.1016/j.ajog.2020.05.003

Temesgen, K., Workie, A., \& Dilnessa, T. (2020). The impact of COVID-19 infection on maternal and reproductive health care services in governmental health institutions of Dessie town, North-East Ethiopia, 2020 G.C. MedRxiv. https://doi.org/10.1101/2020.09.20.20198259

Traylor, C. S., Johnson, J. D., Kimmel, M. C., \& Manuck, T. A. (2020). Expert Review Effects of psychological stress on adverse pregnancy outcomes and nonpharmacologic 
approaches for reduction: an expert review. The American Journal of Obstetrics \& Gynecology MFM, 2(4), 100229. https://doi.org/10.1016/j.ajogmf.2020.100229)

Williamson, H. A., Jr, LeFevre, M., \& Hector, M., Jr. (1989). Association between life stress and serious perinatal complications. The Journal of family practice, 29(5), 489-496.

World Health Organization. (2020). [WHO website] WHO announces COVID-19 outbreak a pandemic. Retrieved from: https://www.euro.who.int/en/health-topics/healthemergencies/coronavirus-covid-19/news/news/2020/3/who-announces-covid-19outbreak-a-pandemic

World Health Organization. (n.d.). [WHO website] WHO Coronavirus Disease (COVID-19) Dashboard. Retrieved from: https://covid19.who.int/

Wu, Y., Zhang, C., Liu, H., Duan, C., Li, C., Fan, J., . . Xu, Y. (2020). Perinatal depressive and anxiety symptoms of pregnant women during the coronavirus disease 2019 outbreak in China. American Journal of Obstetrics and Gynecology, 223(2), 240.e1-240.e9. 